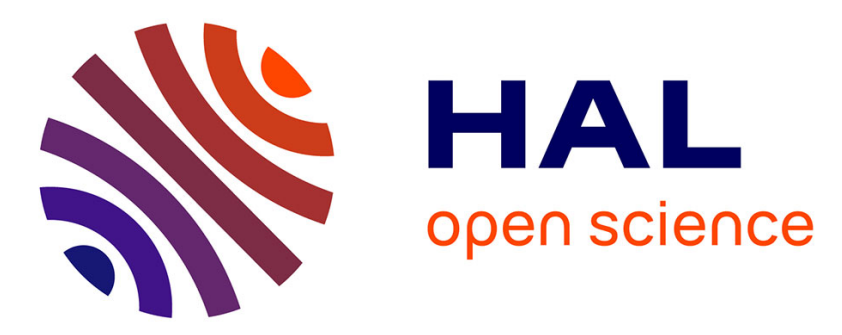

\title{
Effect of graphene layer on the localized surface plasmon resonance (LSPR) and the sensitivity in periodic
}

\section{nanostructure}

\author{
Mohamed El Barghouti, Abdellatif Akjouj, Abdellah Mir
}

\section{To cite this version:}

Mohamed El Barghouti, Abdellatif Akjouj, Abdellah Mir. Effect of graphene layer on the localized surface plasmon resonance (LSPR) and the sensitivity in periodic nanostructure. Photonics and Nanostructures - Fundamentals and Applications, 2018, 31, pp.107-114. 10.1016/j.photonics.2018.06.009 . hal-03183483

\section{HAL Id: hal-03183483 \\ https://hal.science/hal-03183483}

Submitted on 20 Aug 2021

HAL is a multi-disciplinary open access archive for the deposit and dissemination of scientific research documents, whether they are published or not. The documents may come from teaching and research institutions in France or abroad, or from public or private research centers.
L'archive ouverte pluridisciplinaire HAL, est destinée au dépôt et à la diffusion de documents scientifiques de niveau recherche, publiés ou non, émanant des établissements d'enseignement et de recherche français ou étrangers, des laboratoires publics ou privés. 


\title{
Effect of graphene layer on the localized surface plasmon resonance (LSPR) and the sensitivity in periodic nanostructure
}

\author{
Mohamed El barghouti ${ }^{\mathrm{a}, *}$, Abdellatif Akjouj ${ }^{\mathrm{b}}$, Abdellah Mir $^{\mathrm{a}}$ \\ a Laboratory for the Study of Advanced Materials and Applications (LEM2A), Physics Department, Faculty of Science, Moulay Ismail University, B.P. 11201, Zitoune, Meknes, Morocco \\ ${ }^{\mathrm{b}}$ Institute of Electronics, Microelectronics and Nanotechnology, UMR CNRS 8520, Lille University, FST, Department of Physics, 59655 Villeneuve d'Ascq, France
}

\section{Introduction}

As part of our study, we analyze the evolution of the localized surface plasmon resonance (LSPR) that has been extensively studied over the last year $[1,2]$ in a periodic nanostructure 2D metal nanoparticle. We study in this work the influence of a few thin layers of graphene on the LSPR behavior of gold nanoparticles (AuNPs).

The idea is to highlight the role of a few layers of graphene as one of the finest collections. The optical properties are significantly dependent on the chemistry surface $[3,4]$ on which they are deposited, and on the refractive index of the dielectric environment surrounding these nanostructures $[5,6]$. Namely that apart from low graphene layer thicknesses (strong red shift of the resonance), the plasmon oscillation amplitude is increasing almost linearly with up to $5 \mathrm{~nm}$, compared to the case where the gold nanoparticles are directly related to the detection of dielectric, the surface plasmon resonance of AuNPs deposited on a glass $\left(\mathrm{SiO}_{x}\right)$ substrate $\left(n_{1}=1.45\right)$ and covered with layers of a dielectric material [3,7-12].

Numerical simulations of the structure $\left(\mathrm{SiO}_{x} / \mathrm{AuNPs} / \mathrm{Graphene}\right.$ ) $\mathrm{SiO}_{x}$ ) show a high sensitivity of $59.81\left(\mathrm{~nm} \mathrm{RIU}^{-1}\right)$ for the monolayer graphene $0.34 \mathrm{~nm}[7,12]$, and response of plasmonic metal nanoparticles of various geometrical parameters gold nanoparticles, these findings are consistent with other reports in the literature [13] demonstrating long row detection of refractive index on plasmonic nanostructures.

Gold nanoparticles [14] are of great interest for the development of chemical and biological nanosensors and their use in the detection area based on the localized surface plasmon resonance (LSPR) [1,2].

Numerical simulations of our structure indicate that the resonance wavelength of the electric field mode is the surface plasmon, the hot spots of which (areas of high field intensities) are localized to the lower corners of the AuNPs, deferens by compared to the structure $\mathrm{SiO}_{x} /$ AuNPs $/ \mathrm{SiO}_{x}$, published in literature $[13,15]$ and that on $\mathrm{SiO}_{2}$ substrates $[16,17]$.

\section{Theoretical methods}

\subsection{Theoretical model}

The optical properties of gold nanoparticles are solved numerically, in the frequency domain, using the scattered field formulation. Field analysis was performed using a commercially available finite-elementmethod. The simulation method has been well documented in [18-20].

A layer of gold nanoparticles of diameter $(l)$, height $(h)$ and interparticle distance $(a)$, are coated with a graphene thin-layer-thickness; and immersed in a homogeneous matrix, a transparent glass substrate

\footnotetext{
* Corresponding author

E-mail address: Mohamed.elbarghouti@gmail.com (M. El barghouti).
} 
Table 1

Gold (Au) Lorentz-Drude model parameters.

\begin{tabular}{lllll}
\hline Term & $f_{m}[\mathrm{rad} / \mathrm{s}]$ & $\omega_{p}[\mathrm{rad} / \mathrm{s}]$ & $\omega_{m}[\mathrm{rad} / \mathrm{s}]$ & $\Gamma_{m}[\mathrm{rad} / \mathrm{s}]$ \\
\hline$m=0$ & 0.760 & $13.72 \times 10^{15}$ & 0.00 & $0.08052 \times 10^{15}$ \\
$m=1$ & 0.024 & $13.72 \times 10^{15}$ & $0.6305 \times 10^{15}$ & $0.3661 \times 10^{15}$ \\
$m=2$ & 0.010 & $13.72 \times 10^{15}$ & $1.261 \times 10^{15}$ & $0.5241 \times 10^{15}$ \\
$m=3$ & 0.071 & $13.72 \times 10^{15}$ & $4.538 \times 10^{15}$ & $1.322 \times 10^{15}$ \\
$m=4$ & 0.601 & $13.72 \times 10^{15}$ & $6.538 \times 10^{15}$ & $3.789 \times 10^{15}$ \\
$m=5$ & 4.384 & $13.72 \times 10^{15}$ & $20.24 \times 10^{15}$ & $3.364 \times 10^{15}$ \\
\hline
\end{tabular}

of $\mathrm{SiO}_{x}$ (refractive index $n_{1}=1.45$ ). The frequency-dependent complex permittivity of metal (gold) is described by the Lorentz-Drude model [21,22].

$\varepsilon(\omega)=\varepsilon_{r, \infty}+\sum_{m=0}^{M} \frac{f_{m} \omega_{p}^{2}}{\omega_{m}^{2}-\omega^{2}+j \omega \Gamma_{m}}$

where $\varepsilon_{r, \infty}$ are the relative permittivity at infinite frequency, $\omega_{p}$ the plasma frequency, and $\omega_{m}, f_{m}$ and $\Gamma_{m}$ are the resonance frequency, strength and damping frequency, respectively, of $m$ th oscillator. The Lorentz-Drude model uses $\mathrm{M}$ damped harmonic oscillators to describe the small resonances observed in the metal's frequency response. The values of the constants in Eq. (1) are taken from reference [21]. The value of the dielectric constant of infinite frequencies in reference [21] is $\varepsilon_{\infty}=1$. The values of the other L-D parameters are given in Table 1 .

\subsection{Refractive index}

Covering the nanoparticles with a no-absorbent and no-dispersive is not the only option to provide them with chemical protection. Thus, a material as graphene, in addition to protecting the nanoparticles, provides advantages for their sensitivity. It has indeed been shown to intercalate graphene sheets between the metal film and the medium detection delocalized surface plasmon resonance sensors generates an increased sensitivity $[23,24]$. This increase is not only due to the optical properties of graphene, but also to its excellent adsorption properties of biomolecules [25].

The layer of our model is monolayer graphene $(d=0.34 \mathrm{~nm})$ and its complex refractive index $\left(n_{g}\right)$ in the visible range is an absorbent and dispersing material, the refractive index of which is given by the following formula (2) [26]:

$n_{g}=3.0+\frac{C_{1}}{3} \lambda$

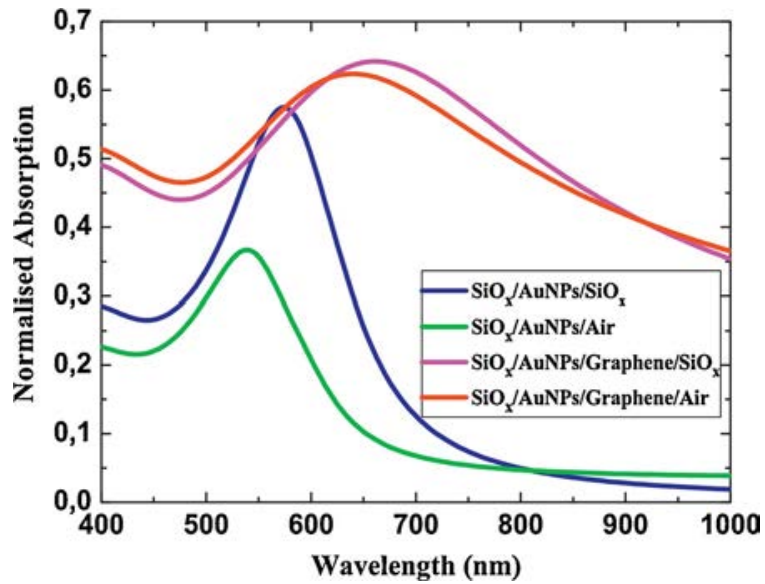

Fig. 2. Computed absorption spectra of AuNPs, in the case where $l=25 \mathrm{~nm}$, $a=80 \mathrm{~nm}, h=20 \mathrm{~nm}$ and the thickness of the graphene layer $(d=5 \mathrm{~nm})$, for two detection Milieus, $\mathrm{SiO}_{x}$ (pink) and air (red). Or without a layer of graphene $(d=0 \mathrm{~nm})$ for $\mathrm{SiO}_{x}$ (blue) and air (green). (For interpretation of the references to color in this figure legend, the reader is referred to the web version of the article.)

where the constant $C_{1}=5.446 \mu \mathrm{m}^{-1}$ [27], and is $\lambda$ the wavelength of the incident light in $\mu \mathrm{m}$.

\section{Results and discussion}

\subsection{The effect of graphene layers deposited on the gold nanoparticles}

Fig. 1 shows schematically the studied system. The network of nanoparticles is characterized by $l=25 \mathrm{~nm}, h=20 \mathrm{~nm}$ and $a=70 \mathrm{~nm}$. It is excited by a plane wave generated in the substrate refractive index $n_{1}=1.45\left(\mathrm{SiO}_{x}\right)$, propagating along the $O y$-axis and the electric field is polarized along the $O x$-axis. Calculating is constructed as to simulate a network infinitely periodic nanoparticles and gold, constituting the nanoparticles is described by the Lorentz-Drude model $[21,22]$.

These values of nanoparticles parameters chosen following the investigation described above, on the basis of their experience [13], show a sectional view of the overall shape of the particles. We can note the rather random shape of the particles, which made it necessary to determine statistics relating to the dimensions of the nanoparticles in the plane (inter-particle diameter and distance), $l$ and $p=a-l$ (respectively). The results of such statistics, respectively for $l$ and $p$, are

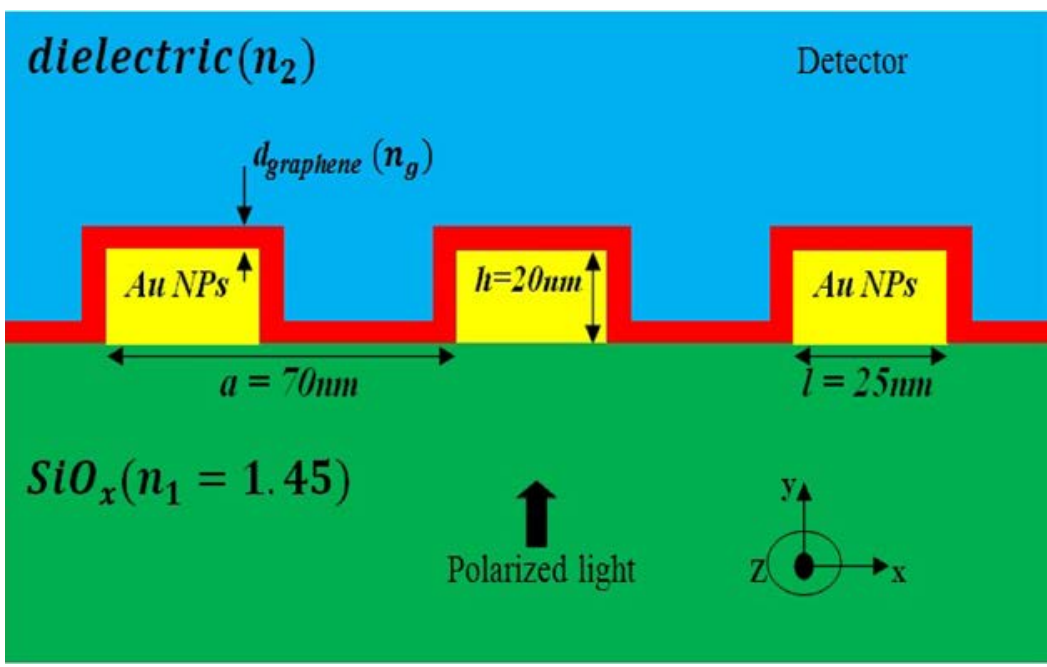

Fig. 1. Typical diagram of the modeled structure, namely gold ribbons apart and characterized by $a$ length $l$ and height $h$. the tapes are covered with a thin layer of graphene. 

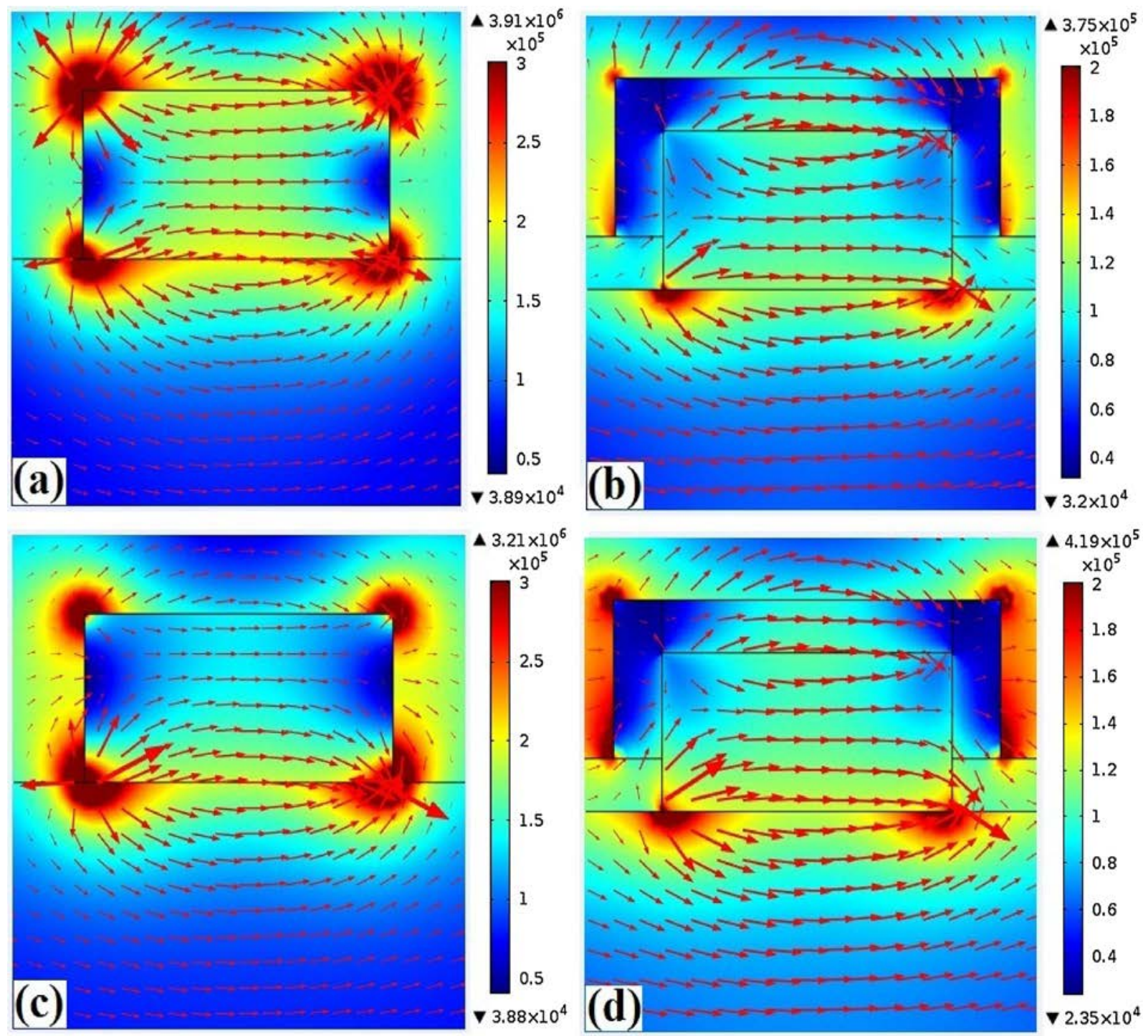

Fig. 3. Maps of the electric field for monochromatic incident radiation at the wavelength of maximum absorption as calculated by numerical analysis of metal studs, on (a) $\mathrm{SiO}_{x} / \mathrm{AuNPs} / \mathrm{SiO}_{x}$ at $\lambda_{\max }=574.71 \mathrm{~nm}$, (b) $\mathrm{SiO}_{x} / \mathrm{AuNPs} / \mathrm{Graphene} / \mathrm{SiO}_{x}$ at $\lambda_{\max }=657.90 \mathrm{~nm}$, (c) $\mathrm{SiO}_{x} / \mathrm{AuNPs} / \mathrm{Air}$ at $\lambda_{\max }=537.63 \mathrm{~nm}$, and (d) SiO ${ }_{x} / \mathrm{AuNPs} /$ Graphene/Air at $\lambda_{\max }=636.94 \mathrm{~nm}$, where $l=25 \mathrm{~nm}, a=80 \mathrm{~nm}$ and $h=20 \mathrm{~nm}$, and that for the thickness value graphene $d=5 \mathrm{~nm}$.

obtained thanks to SEM [13]. We then see that the most probable values are $25 \pm 8 \mathrm{~nm}$ for the diameter $l$, and $16 \pm 8 \mathrm{~nm}$ for the inter-particle distance $p$. For the third dimension which is the height $h$ of the values obtained is $13.6 \pm 3 \mathrm{~nm}$ [13]. To do this, the thickness of the layer of the dielectric (graphene) deposited on the particles at $d=5 \mathrm{~nm}$ was fixed on the one hand; and on the other hand the length $l$ (Fig. 9), the grating period of the particles $a$, and then the height of the pads $h$ have been varied. These variations were made with the experimental constraints, a one-dimensional filling ratio for the theoretical structure $l$, $a=0.37$; and the choice of the values of $l$, a $(a=l+p)$ and $h$ in the same orders of quantities provided by the experiment $[13,15]$. The effect of the nanoparticles length in the plane of the substrate supporting them $l$, on the nanostructure plasmonic response was looked at keeping the other geometrical parameters constant, that is to say $a=70 \mathrm{~nm}$, $h=20 \mathrm{~nm}$ and $d=5 \mathrm{~nm}$. In Fig. 9, there is a fairly large displacement of the wavelength corresponding to the plasmonic response of the nanoparticles to the long wavelengths (low frequencies). This is done by increasing progressively from $15 \mathrm{~nm}$ to $55 \mathrm{~nm}$. There is also a higher absorption of the pads with the increase of $l$. This can be related, from one side, to more confined energy with the increase of the particle-dielectric interfaces at resonance, from the other side, the increase of the nanoparticles. Also, we have seen previously that in high interAuNPs coupling regime $[15,28,29]$. There is also a sharp decrease in the width of the resonances when the nanoparticles are distant from each other. The widening of the resonances results from the fact that the studied system becomes more and more close to a system composed of a metallic film when the width of the nanoparticles is increased while keeping the period of the constant network.

Metallic gold nanoparticles characterized by the geometrical parameters, $(l)$ to the diameter $(h)$ to the height, and $(a)$ the grating period of the nanoparticles, the nanoparticles are coated with a layer of graphene to thin thickness $(d)$ in the context of this study, the refractive index graphene $n_{g}$, and the whole is immersed in a homogeneous matrix of $\mathrm{SiO}_{x}$ (refractive index $n_{1}=1.45$ ).

Comparing the results of the absorption spectrum calculated with the L-D model ( $d=5 \mathrm{~nm}$ thick graphene layer) with the absorption spectrum of graphene results without $(d=0)$ that you had already published [13] to the same structure.

We recall that in the frequency range of this study, we get the more given graphene layer that gives without graphene.

Fig. 2 shows the theoretical calculation results of the normalized absorption of the metal pins (structure $\mathrm{SiO}_{x} / \mathrm{AuNPs} / \mathrm{Graphene}_{\mathrm{SiO}}$ ) as 

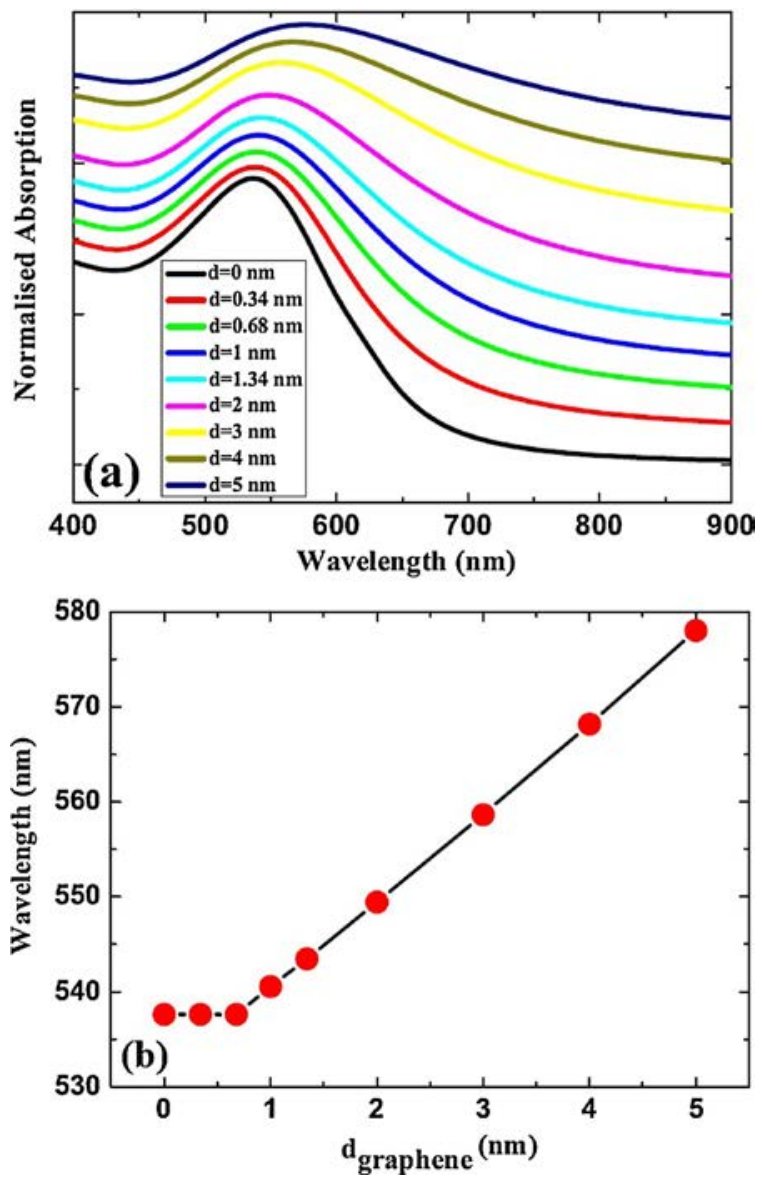

Fig. 4. (a) Change of absorption spectra versus the wavelength of $\mathrm{SiO}_{x} / \mathrm{AuNPs} /$ Graphene/ $/ \mathrm{SiO}_{x}$. The absorption is calculated for AuNPs of different thickness of a graphene $d$. The period of AuNPs is fixed at $a=70 \mathrm{~nm}$, the height of AuNPs is fixed at $h=20 \mathrm{~nm}$ and the length of AuNPs at $l=25 \mathrm{~nm}$. (b) Evolution of LSPR peak with change in graphene film thickness $d . a=70 \mathrm{~nm}, l=25 \mathrm{~nm}$ and $h=20 \mathrm{~nm}$.

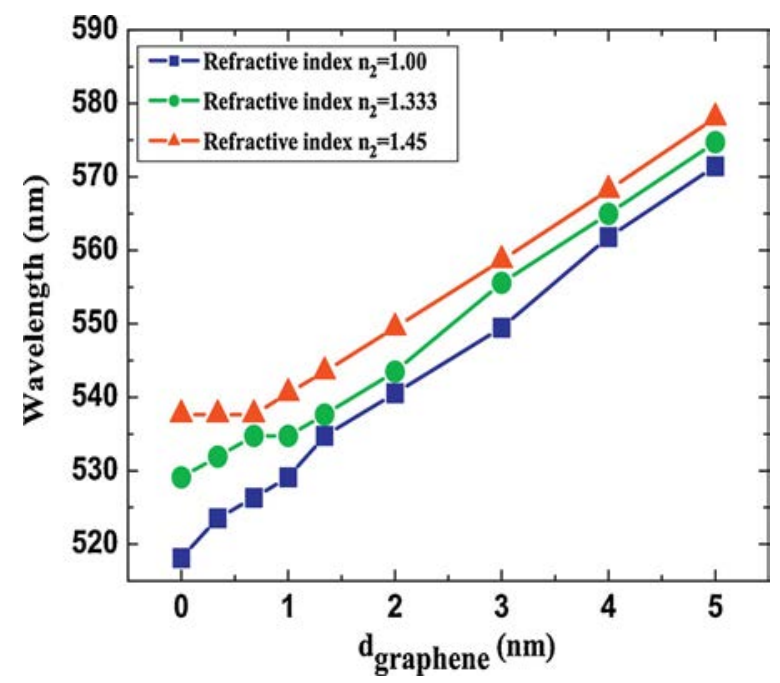

Fig. 5. Shifting the LSPR peak wavelength depending on the refractive index of the surrounding medium, air (blue square), water (green circle) and $\mathrm{SiO}_{\mathrm{x}}$ (red triangle). (For interpretation of the references to color in this figure legend, the reader is referred to the web version of the article.) a function of frequency, and the result of the interaction of the studs with a light pulse incident $[400-1000 \mathrm{~nm}]$ the values of the structural parameters are chosen: $l=30 \mathrm{~nm}, a=80 \mathrm{~nm}$ and $h=15 \mathrm{~nm}$.

In order to compare the results calculated with the layer of graphene and graphene-free, we can see a pretty good increase between the theoretical spectra with and without graphene layer, there has been a dramatic decrease in the resonance wavelength of the plasmon studs passing from $636.94 \mathrm{~nm}$ observed on $\mathrm{SiO}_{x} /$ AuNPs/Graphene (red line) to $537.63 \mathrm{~nm}$ observed on $\mathrm{SiO}_{x} /$ AuNPs (green line) (with an energy decay absorbed 0.62 to 0.37 ), for a thickness of the air detection medium. In the case where the pads (metal particles) are covered with 5 $\mathrm{nm}$ of graphene (pink line), as in the case of pads uncovered graphene (blue lines) with thickness $\mathrm{SiO}_{x}$ detection medium. There has been a dramatic decrease in the resonance wavelength of the plasmon studs passing from $657.90 \mathrm{~nm}$ on $\mathrm{SiO}_{x} /$ AuNPs/Graphene to $574.71 \mathrm{~nm}$ on $\mathrm{SiO}_{x} /$ AuNPs (with a decrease of energy absorb from 0.64 to 0.57 ).

Furthermore, beyond the comparison results, with and without a layer of graphene, it is noted that in both there is cases a strong absorption of the studs to the $574.71 \mathrm{~nm}$ wavelength (without the graphene layer) and $657.90 \mathrm{~nm}$ when those are covered $(d=5 \mathrm{~nm})$ to ( $h=15 \mathrm{~nm}, a=80 \mathrm{~nm}$, and $l=30 \mathrm{~nm}$ ). These wavelengths correspond to the excitation of the plasmon-polariton mode particle surface, confined at the interface between the particles and the surrounding dielectric.

Besides, in Fig. 2 are the absorption spectra of the system studied for different cases without graphene layer and with the graphene layer $(5 \mathrm{~nm})$. It is observed that the addition of graphene generates a loss of symmetry of the resonance bands due to their widening for the large wavelengths $\left(\lambda>\lambda_{\max }\right)$. This enlargement arises from the fact that the absorption of graphene increases with the wavelength $\left(n_{g}=3.0+\frac{C_{1}}{3} \lambda\right.$, $C_{1}=5.446 \mu \mathrm{m}^{-1}$ ) [26]. This is important for finding plasmon sensors with optimum properties. Indeed, sensitivity performance is not only correlated with a strong spectral shift in resonance due to a refractive index variation. They are also dependent on the spectral width of the resonance bands, since it is easier to spot the movement of the latter when they are fine.

The excited electrical field distributions on resonance, at a normal incidence structures, are represented by cards of electric field in Fig. 3(a-d), which indicate that this mode is a dipolar localized surface plasmon, whose hot spots (areas of high field strengths) are pushed to the top and lower corners of the AuNPs of the structure lacking the Graphene layer, and to lower corners of higher AuNPs graphene layer of the structure with graphene (Fig. 3a). The confined fields within the corners of the structure AuNPs of $\mathrm{SiO}_{x} / \mathrm{AuNPs} / \mathrm{SiO}_{x}$ with a strong localization on the lower surface of the AuNPs, compared to the structure AuNPs/SiO $/$ /Air in (Fig. 3c) in which a strong location is observed on the upper and lower corners of the AuNPs [30,31].

For the structure $\mathrm{SiO}_{x} / \mathrm{AuNPs} / \mathrm{Graphene} / \mathrm{SiO}_{x}$ in Fig. $3 \mathrm{~b}$ the fields are, basically, limited to the space between the graphene layer and the dielectric of detection which are lower than the AuNPs. It can be seen by comparing Fig. 3(a and b) that when the nanoparticles covered by 5 $\mathrm{nm}$ of graphene have hot spots at each of their corners and that the two hot spots in contact with the substrate have spread spatially. Moreover, the addition of graphene has resulted in an increase in the amplitude of the electric field in the interparticle space followed by a decrease in peak value intensity. This observed decrease in field strength is due to the coupling between the plasmon mode and the Fabry-Perot cavity modes connected to the presence of the graphene and $\mathrm{SiO}_{x}$ layer presented above the gold nanoparticles [28,31-33].

In the structure (Fig. 3d) $\mathrm{SiO}_{x} /$ AuNPs/Graphene/Air fields are supposed to have a change in the lower refractive index. On the one hand, the fields are partially confined to the dielectric detection and inside the AuNPs. On the other hand, this structure shows a lack of field distribution at the place where the top corners of the graphene bound in the middle of detection, and therefore, at the interface layer with air. 
Table 2

Simulated wavelength and sensitivity to gold nanoparticles for different graphene layer thicknesses of structure $\mathrm{SiO}_{x} / \mathrm{AuNPs} / \mathrm{Graphene} / \mathrm{SiO}_{x}$, other parameters are kept constant $l=25 \mathrm{~nm}, h=20 \mathrm{~nm}$ and $a=70 \mathrm{~nm}$.

\begin{tabular}{llll}
\hline $\begin{array}{l}\text { Graphene thickness }(d) \\
(\mathrm{nm})\end{array}$ & $\lambda_{\max }(\mathrm{nm})$ & $\begin{array}{l}\text { Normalised } \\
\text { absorption }\end{array}$ & $S=\frac{\Delta \lambda_{\max }}{\Delta n_{2}}(n m / R I U)$ \\
\hline 0 & 537.63 & 0.38 & 32.93 \\
0.34 & 537.63 & 0.38 & 59.81 \\
0.68 & 537.63 & 0.38 & 26.47 \\
1 & 540.54 & 0.39 & 39.92 \\
1.34 & 543.48 & 0.40 & 8.92 \\
2 & 549.45 & 0.41 & 41.60 \\
3 & 558.66 & 0.43 & 36.31 \\
4 & 568.18 & 0.44 & 15.10 \\
5 & 578.03 & 0.45 & 23.76 \\
\hline
\end{tabular}

The lowest optical index of air $\left(n_{2}=1\right)$ compared to higher graphene $\left(n_{g}\right)$ explains why this mode shows a lower location of the electric field in comparison with similar manufactured networks in mid-detection $\mathrm{SiO}_{x}\left(n_{1}=1.45\right)$ [33-36].

In this study, the effect of the graphene layer, registered on gold nanoparticles was studied by gradually increasing the thickness of the graphene layers of $d=0.34 \mathrm{~nm}$ at $5 \mathrm{~nm}$.

Fig. 4a shows the range of absorption of the plots in function. The gold nanoparticles of the values of $d=0.34,0.68,1,1.34,2,3,4$, and $5 \mathrm{~nm}$, keeping all other parameters constant $(a=70 \mathrm{~nm}, h=20 \mathrm{~nm}$ and $l=25 \mathrm{~nm})$. There is an absorption $0.38(d=0.34 \mathrm{~nm})$ to 0.56 $(d=5 \mathrm{~nm})$.

To better understand this oscillation observed in Fig. 4a, the wavelength plotted plasmon response as a function of thickness $(d)$ of graphene layer, which has been calculated theoretically with the Drude-Lorentz model [20] (Fig. 4b), Shows a significant linear increase in the resonant wavelength plasmon LSPR with the thickness of layer of
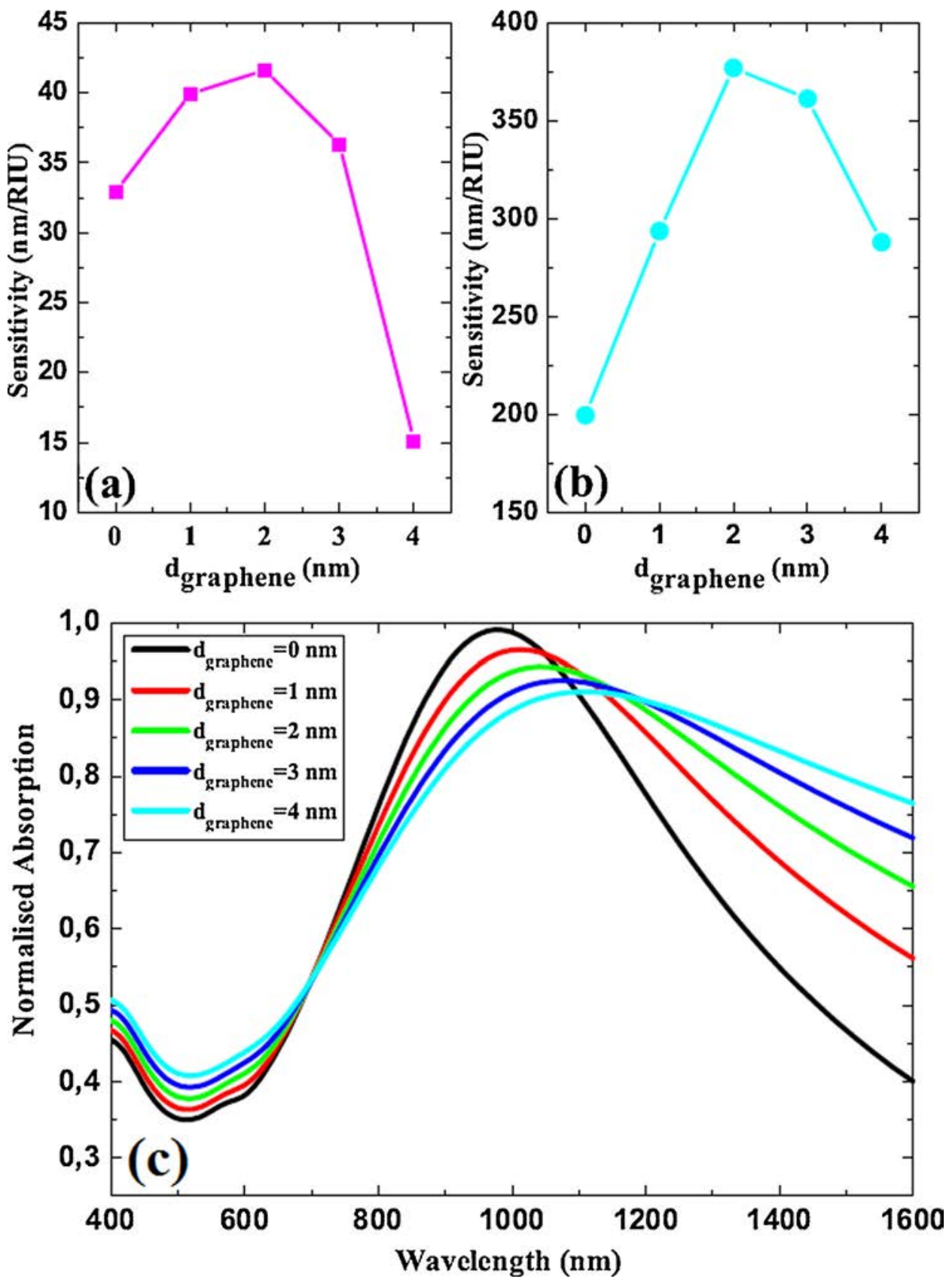

Fig. 6. Evolution of the sensitivity and absorption spectrum of the system studied according to the thickness of graphene covering the nanoparticles of gold. (a) Nanowires are characterized by $l=25 \mathrm{~nm}, h=20 \mathrm{~nm}$ and $a=70 \mathrm{~nm}$, the refractive index of the substrate on which they are deposited is $n_{1}=1.45$. The sensitivity was approximated by $n_{2}$ is the refractive index of the detection medium. The two media used to determine the sensitivity were indices $n_{2}=1.000$ and 1.45 . (b and c) The nanowires are characterized by $l=100 \mathrm{~nm}$, $h=15 \mathrm{~nm}$ and $a=125 \mathrm{~nm}$, the refractive index of the substrate on which they are deposited is $n_{1}=2.00$ (ITO), the two media used to determine the sensitivity have $n_{2}=1.000$ and 1.333 . 

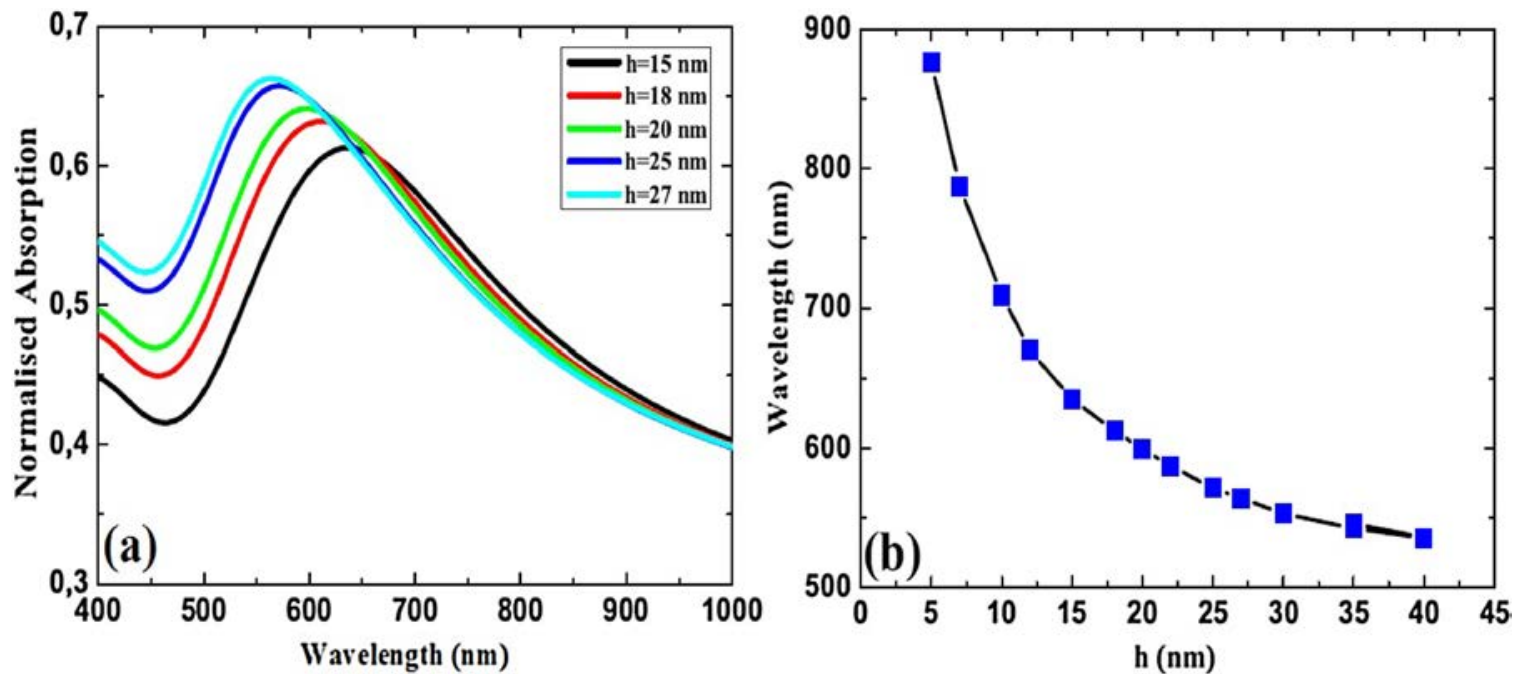

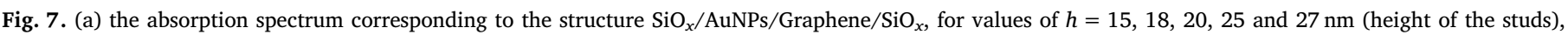

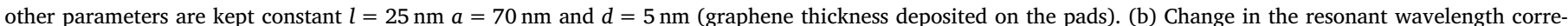
sponding to the absorption observed in (a), according to the height $h$ of the metal pads on a wider range of values.
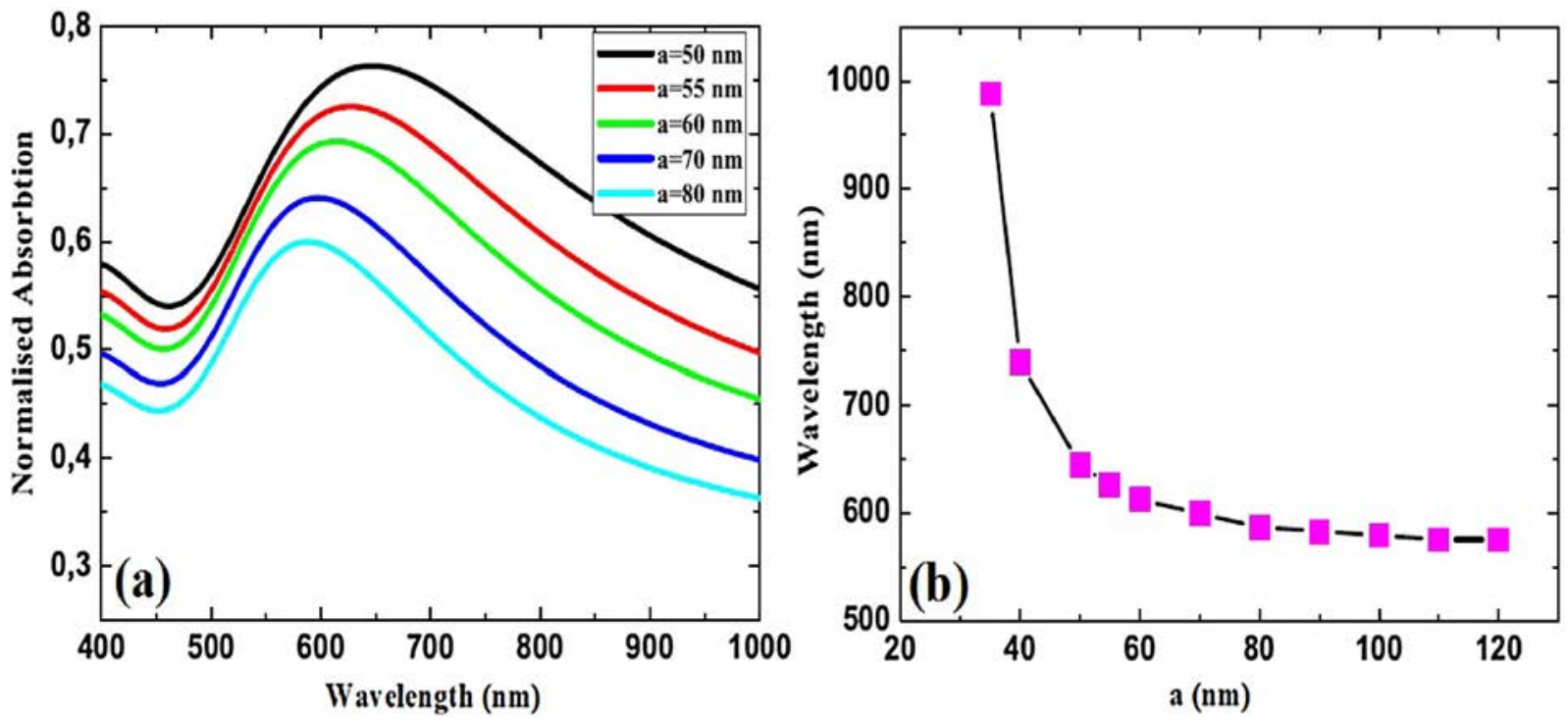

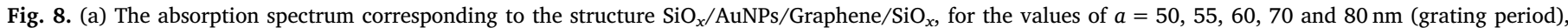

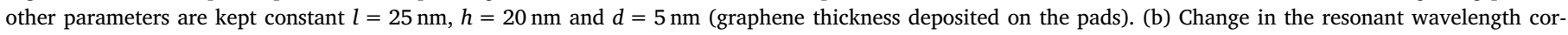
responding to the absorption observed in (a), depending on the period of the array of metal contacts $a$, over a wider range of values.

graphene of $537.63 \mathrm{~nm}$ at $d=0.68 \mathrm{~nm}$ to $578 \mathrm{~nm}$ at $d=5 \mathrm{~nm}$.

Indeed, the wavelength remains constant at $537.63 \mathrm{~nm}$ for the starting value of the resonance wavelength on the curve representing LSPR does not depend on the graphene layer $(d=0 \mathrm{~nm})$, and for $d=0.34 \mathrm{~nm}$ (single layer) and $d=0.68 \mathrm{~nm}$ (bi-layer). The increase of $(0.68 \mathrm{~nm} \leqslant d \leqslant 5 \mathrm{~nm})$ is solely due to the red shift of LSPR when keeping the other parameters constant nanoparticles.

The effect of the refractive index of the interface $\mathrm{SiO}_{x} /$ AuNPs/ Graphene was studied by recording the wavelength shift when it is changed, in different proportions giving the refractive indices ( $n_{2}=1.00$ square symbols for air, $n_{2}=1.333$ round symbols for water, and $\mathrm{SiO}_{x} n_{2}=1.45$ triangular symbols), the response of LSPR spectrum is usually described by Fig. 5 shows that the position of $\lambda_{\max }$ moves to higher wavelengths with an increase in the refractive index. The variation of the position of $\lambda_{\max }$, shows a linear dependence in accordance with the refractive index of the surrounding medium. Sensitivity, defined as the ratio of the variation of the Plasmon's position band in the variation of the refractive index [28,37].
$S=\frac{\Delta \lambda_{\max }}{\Delta n_{2}}\left(\mathrm{~nm} \mathrm{RIU}^{-1}\right)$

(Nanometer change unit by refractive index) is determined from the slope of Fig. 5 and increases if the thickness of the graphene layer interface decreases plasmonic (Table 2).

Fixing the length of particles $l=25 \mathrm{~nm}$, the grating period $a=70 \mathrm{~nm}$ and height $h=20 \mathrm{~nm}$ particles and then varying the refractive index $n_{2}$ of the dielectric layers deposited on the thinnest layer of the graphene.

Fig. 5 shows the change in resonance wavelength as a function of the thickness of graphene for different detection media $n_{2}=1.00$ (air), $n_{2}=1.333$ (water), and $n_{2}=1.45\left(\mathrm{SiO}_{x}\right)$. There is the offset of the resonance wavelength LSPR $\left(\lambda_{\max }\right)$ for a same variation of $n_{2}$ is different according to the value of $d$. For $d=0.34 \mathrm{~nm}$ is noted that the resonance wavelength plasmon particles as a function of refractive index is $526 \mathrm{~nm}$ (air), $539 \mathrm{~nm}$ (water) and $546 \mathrm{~nm}\left(\mathrm{SiO}_{x}\right)$. It is observed in Table 2, the sensitivity changes when going from $d=0 \mathrm{~nm}$ to $d=5 \mathrm{~nm}$. 

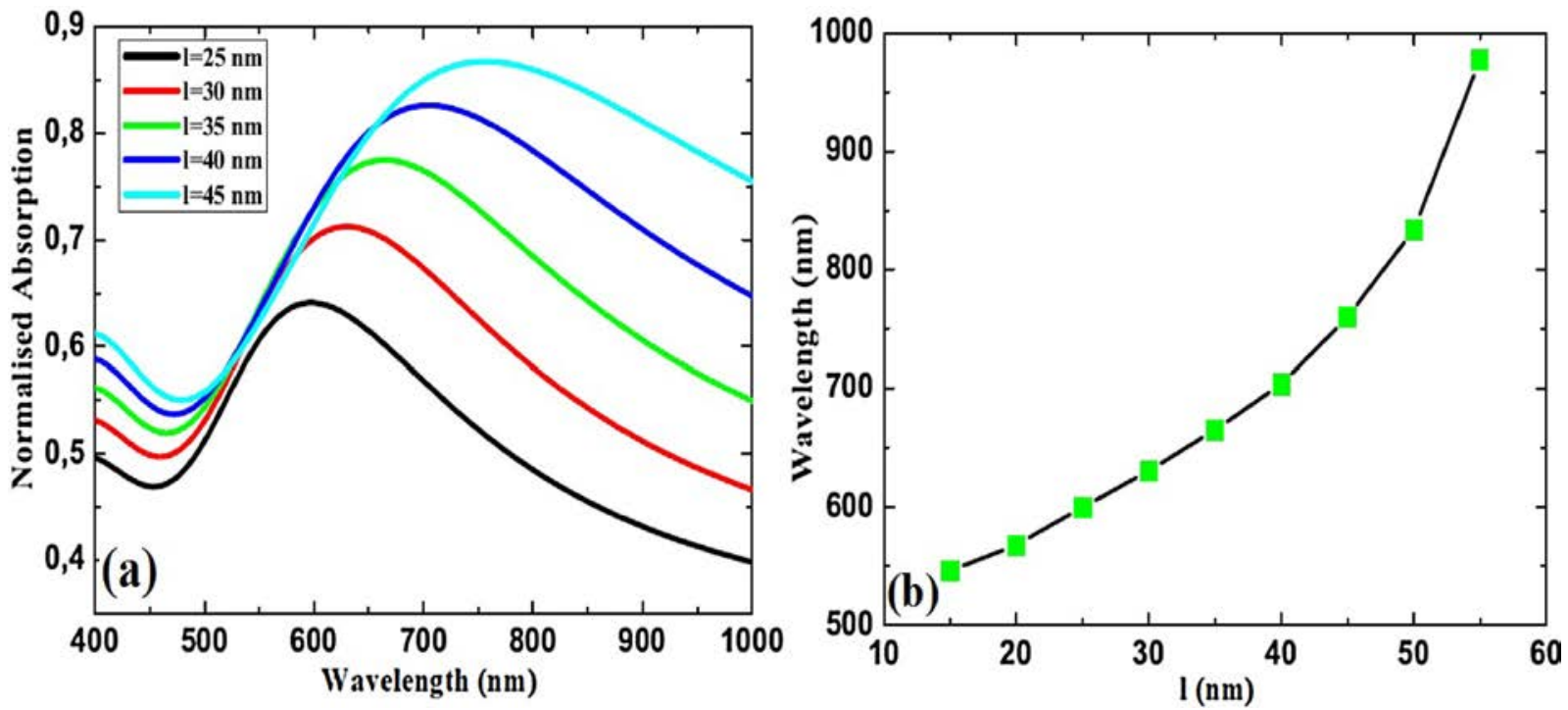

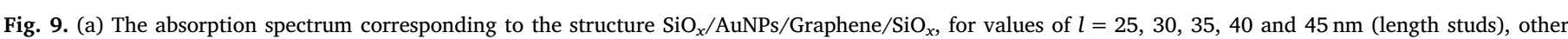

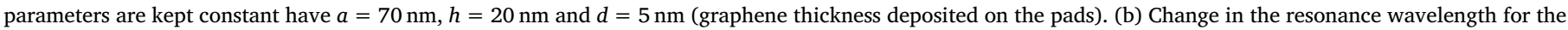
absorption observed in (a), depending on the length of the metal pads $l$ on a wider range of values.

Indeed, $S=32.93 \mathrm{~nm} / \mathrm{RIU}$ for $d=0 \mathrm{~nm}$ and $S=59.81 \mathrm{~nm} / \mathrm{RIU}$ for $d=0.34 \mathrm{~nm}$.

Adding that in the case of depositing thinner thickness of graphene, there is a strong shift towards the red resonance, plasmon oscillation's amplitude increases almost linearly with $d$. Furthermore, it was explained that the sensitivity of nano-sensors based on this phenomenon is related to the displacement of the resonance wavelength of the plasmon response of the particles with $d$, that is to say in the regions of $d$ where the variation of the oscillation amplitude is large, the sensitivity is optimal. This indicates a greater effectiveness of bio-detection with these structures.

The objective of this part is to study quantitatively using the FEM method, the influence of refractive indices $n_{1}$ and $n_{2}$ on the oscillation of the sensitivity of nanostructure to the plasmon resonance when $d$ (graphene) varies. To do this, we start from the same system as before. The curve representing the evolution of the sensitivity (Eq. (3)) According to the thickness $(d)$ can be characterized by the two nanostructures defined in Fig. 6:

In a second phase, several numerical simulations were carried out to determine how the sensitivity evolves according to the thickness of graphene deposited on the nanoparticles. The results obtained appear in Fig. 6. First, an increase in sensitivity is observed for graphene thicknesses between 0 and $2 \mathrm{~nm}$ for both nanostructures.

In fact, (Fig. 6a) $S=32.92 \mathrm{~nm} /$ RIU for $d=0 \mathrm{~nm}$ and $S=41.6 \mathrm{~nm} /$ RIU for $d=2 \mathrm{~nm}$, corresponding to an increase of about $26.37 \%$. Fig. $6 \mathrm{~b}, S=199.76 \mathrm{~nm} / \mathrm{RIU}$ for $d=0 \mathrm{~nm}$ and $S=376.85 \mathrm{~nm} / \mathrm{RIU}$ for $d=2 \mathrm{~nm}$, corresponding to an increase of about $88.65 \%$. Then the sensitivity decreases from $d=2 \mathrm{~nm}$, to reach $15.1 \mathrm{~nm} / \mathrm{RIU}$ (Fig. 6a) and $287.96 \mathrm{~nm} /$ RIU (Fig. 6b) When $d=4 \mathrm{~nm}$.

Fig. $6 \mathrm{c}$ represents the absorption spectra of the system studied for different thicknesses of graphene. It is observed that the addition of graphene generates a loss of symmetry of the resonance bands due to their widening for large wavelengths $\left(\lambda>\lambda_{\max }\right)$. This enlargement arises from the fact that the absorption of the graphene increases with the wavelength. This is important for finding a plasmon sensor with optimum properties. Indeed, sensitivity performance is not only correlated with a strong spectral shift in resonance due to a refractive index variation. They are also dependent on the geometric parameters of the gold nanoparticles ( $h, l$ and $a$ ) and the refractive index $\left(n_{1}\right.$ and $n_{2}$ ).

\subsection{Study of plasmonic response of metal particles as a function of geometric parameters of the gold ribbons, $a, l$ and $h$}

The behavior of the optical response of the particles, in particular, the plasmon resonance according to the parameters $l, a$, and $h$. The procedure was as follows: we first fixed the particle length $l=25 \mathrm{~nm}$, and the grating period $a=70 \mathrm{~nm}$, then varied the height $h$ of the particles between 15 and $27 \mathrm{~nm}$.

Fig. 7a gives the absorption spectrum calculated for several values of $h=15,18,20,25$ and $27 \mathrm{~nm}$, a thickness of $5 \mathrm{~nm}$ deposited graphene. Fig. $7 \mathrm{~b}$ shows the change in the resonance wavelength of the plasmon of the particles in terms of $h$, in a wider range of possible values between 5 and $40 \mathrm{~nm}$.

This is a spectacular non-linear decay of the dabs plasmon resonance wavelength that goes from $876.71 \mathrm{~nm}$ to $535.56 \mathrm{~nm}$ (for a displacement of $341.15 \mathrm{~nm}$ ), where h varies only from $5 \mathrm{~nm}$ to $40 \mathrm{~nm}$.

This corresponds with the frequency at which the incident wave is strongly couples the free electrons of the particles, at the interface between the surface of the nanoparticles covering dielectric (graphene). That is to say, gold particle surface plasmon-polariton mode. The wavelength corresponding to this resonance is greatly shifted to the low lengths (toward the blue) wavelength with increasing $h$.

Second, plasmon mode behavior was studied according to the particle network period, and by setting all other parameters $(l=25 \mathrm{~nm}$, $h=20 \mathrm{~nm}$ and $d=5 \mathrm{~nm}$ ). Fig. 8a shows the study of the plasmonic response of the pads according to $a$. In this figure, the absorption spectrum is calculated $a=50,55,60,70$ and $80 \mathrm{~nm}$.

In Fig. $8 \mathrm{~b}$, is carried changing the resonant wavelength of the plasmon pads, depending $a$. The lattice parameter was varied between $35 \mathrm{~nm}$ and $120 \mathrm{~nm}$. There has been a dramatic non-linear decrease in the resonant wavelength plasmon studs passing from $988.41 \mathrm{~nm}$ to $575.28 \mathrm{~nm}$ (for a displacement of $413.13 \mathrm{~nm}$ ), where $a$ is only $35 \mathrm{~nm}$ to $120 \mathrm{~nm}$.

For $a<50 \mathrm{~nm}$, the resonance wavelength increases greatly due to the coupling of the nanoparticles by their evanescent fields (increased confinement of the electromagnetic field in the space inter-ribbons). On the other hand, for $a>110 \mathrm{~nm}$ resonance wavelength hardly varies. Ribbons are far enough apart so that their evanescent fields will almost cover more and tends to the plasmonic response of a single tape.

Third, plasmon mode behavior was studied according to the length of the tape, keeping constant the other geometric parameters, that is to 
say $a=70 \mathrm{~nm}, h=20 \mathrm{~nm}$ and $d=5 \mathrm{~nm}$. In Fig. 9a, it gives the absorption spectrum for $l=25,30,35,40$, and $45 \mathrm{~nm}$.

This is a significant displacement of the wavelength of absorption corresponding to reply Plasmon of the studs to the long wavelength (low frequencies) from 545.84 to $977 \mathrm{~nm}$.

In Fig. 9b, it is reported the development of the frequency of the absorption zeros, observed in Fig. 9a (plasmon response), according to the $l$. in a wider range of possible values between $l=15 \mathrm{~nm}$ and $l=55 \mathrm{~nm}$. This figure shows more precise information on the behavior of the plasmon $l$. It can be seen that between $l=15 \mathrm{~nm}$ and $l=45 \mathrm{~nm}$, that is to say between a situation where the inter-distance blocks is $55 \mathrm{~nm}$ and $25 \mathrm{~nm}$ respectively; the resonance wavelength increases almost linearly with $l$. Starting, of $l=45 \mathrm{~nm}$, there is an abrupt nonlinear growth very important to the resonance wavelength plasmonic, up from $760 \mathrm{~nm}$ to $977 \mathrm{~nm}$ (near infrared), an increase of $10 \mathrm{~nm}$ only. These two points may be explained in part by the fact that between $l=15 \mathrm{~nm}$ and $l=45 \mathrm{~nm}$. When the nanoparticles are weakly coupled, it results in a linear variation with LSPR. While that from $l=45 \mathrm{~nm}$, when the nanoparticles are strongly coupled, and therefore, a non-linear variation of LSPR depending on $l$.

\section{Conclusion}

We have theoretically demonstrated the structural effect of gold nanoparticles covered with thin layers of graphene [0.34 nm, $5 \mathrm{~nm}$ ] on the optical properties. The Lorentz-Drude model was used to calculate the optical signal plasmon resonances localized surface (LSPR), gold nanostructures (AuNPs) on the glass and covered with a thin dielectric layer of graphene and $\mathrm{SiO}_{x}$, indicates that this study provides a better understanding of systems based on resonant metal nanoparticles coupled with graphene layers. To validate the model, we compared the spectrum obtained through numerical simulations of LSPR to those previously released without a layer of graphene. From a practical standpoint, it opens the door to nanotechnology in a controlled and predictable manner. The spectral properties of systems based on metalgraphene nanoparticles to enhance their particular applicability for sensing applications. This study paves the way to highly sensitive sensors and improved biosensors.

\section{References}

[1] K.M. Mayer, J.H. Hafner, Localized surface plasmon resonance sensors, Chem. Rev. 111 (6) (2011) 3828-3857.

[2] S. Szunerits, R. Boukherroub, Sensing using localised surface plasmon resonance sensors, Chemical Communications 48 (72) (2012) 8999-9010.

[3] S. Szunerits, M.R. Das, R. Boukherroub, Short-and long-range sensing on gold nanostructures, deposited on glass, coated with silicon oxide films of different thicknesses, J. Phys. Chem. C 112 (22) (2008) 8239-8243.

[4] I. Doron-Mor, Z. Barkay, N. Filip-Granit, A. Vaskevich, I. Rubinstein, Ultrathin gold island films on silanized glass. Morphology and optical properties, Chem. Mater. 16 (18) (2004) 3476-3483.

[5] M.D. Malinsky, K.L. Kelly, G.C. Schatz, R.P. Van Duyne, Chain length dependence and sensing capabilities of the localized surface plasmon resonance of silver nanoparticles chemically modified with alkanethiol self-assembled monolayers, J. Am. Chem. Soc. 123 (7) (2001) 1471-1482.

[6] J. Zhao, L. Jensen, J. Sung, S. Zou, G.C. Schatz, R.P. Van Duyne, Interaction of plasmon and molecular resonances for rhodamine $6 \mathrm{~g}$ adsorbed on silver nanoparticles, J. Am. Chem. Soc. 129 (24) (2007) 7647-7656.

[7] A.J. Haes, S. Zou, G.C. Schatz, R.P. Van Duyne, Nanoscale optical biosensor: short range distance dependence of the localized surface plasmon resonance of noble metal nanoparticles, J. Phys. Chem. B 108 (22) (2004) 6961-6968.

[8] H. Xu, M. Käll, Modeling the optical response of nanoparticle-based surface plasmon resonance sensors, Sens. Actuators B: Chem. 87 (2) (2002) 244-249.

[9] K.A. Willets, W.P. Hall, L.J. Sherry, X. Zhang, J. Zhao, R.P. Van Duyne, Nanoscale Localized Surface Plasmon Resonance Biosensors. Nanobiotechnology II: More Concepts and Applications, (2007), pp. 159-173.

[10] W.A. Murray, J.R. Suckling, W.L. Barnes, Overlayers on silver nanotriangles: field confinement and spectral position of localized surface plasmon resonances, Nano Lett. 6 (8) (2006) 1772-1777.
[11] T. Rindzevicius, Y. Alaverdyan, M. Käll, W.A. Murray, W.L. Barnes, Long-range refractive index sensing using plasmonic nanostructures, J. Phys. Chem. C 111 (32) (2007) 11806-11810.

[12] A. Gupta, G. Chen, P. Joshi, S. Tadigadapa, P. Eklund, Raman scattering from highfrequency phonons in supported n-graphene layer films, Nano Lett. 6 (12) (2006) 2667-2673.

[13] E. Galopin, A. Noual, J. Niedziółka-Jönsson, M. Jönsson-Niedziółka, A. Akjouj, Y. Pennec, B. Djafari-Rouhani, R. Boukherroub, S. Szunerits, Short-and long-range sensing using plasmonic nanostrucures: experimental and theoretical studies, J. Phys. Chem. C 113 (36) (2009) 15921-15927.

[14] L. Wu, H. Chu, W. Koh, E. Li, Highly sensitive graphene biosensors based on surface plasmon resonance, Opt. Express 18 (14) (2010) 14395-14400.

[15] T. Maurer, R. Nicolas, G. Lévêque, P. Subramanian, J. Proust, J. Béal, S. Schuermans, J.-P. Vilcot, Z. Herro, M. Kazan, et al., Enhancing LSPR sensitivity of au gratings through graphene coupling to au film, Plasmonics 9 (3) (2014) $507-512$.

[16] P. Viste, J. Plain, R. Jaffiol, A. Vial, P.M. Adam, P. Royer, Enhancement and quenching regimes in metal-semiconductor hybrid optical nanosources, ACS Nano 4 (2) (2010) 759-764.

[17] T. Hutter, S.R. Elliott, S. Mahajan, Interaction of metallic nanoparticles with dielectric substrates: effect of optical constants, Nanotechnology 24 (3) (2013) 035201 .

[18] M.W. Knight, N.J. Halas, Nanoshells to nanoeggs to nanocups: optical properties of reduced symmetry core-shell nanoparticles beyond the quasistatic limit, N. J. Phys. 10 (10) (2008) 105006.

[19] D.Y. Lei, A.I. Fernandez-Dominguez, Y. Sonnefraud, K. Appavoo, R.F. Haglund Jr., J.B. Pendry, S.A. Maier, Revealing plasmonic gap modes in particle-on-film systems using dark-field spectroscopy, ACS Nano 6 (2) (2012) 1380-1386.

[20] Y. Zhan, D.Y. Lei, X. Li, S.A. Maier, Plasmonic fano resonances in nanohole quadrumers for ultra-sensitive refractive index sensing, Nanoscale 6 (9) (2014) 4705-4715.

[21] F.-C.F. Tsai, C.J. OBrien, N.S. Petrović, A.D. Rakić, Analysis of optical channel cross talk for free-space optical interconnects in the presence of higher-order transverse modes, Appl. Opt. 44 (30) (2005) 6380-6387.

[22] C. Powell, Analysis of optical-and inelastic-electron-scattering data. II. Application to Al, JOSA 60 (1) (1970) 78-93.

[23] R. Verma, B.D. Gupta, R. Jha, Sensitivity enhancement of a surface plasmon resonance based biomolecules sensor using graphene and silicon layers, Sens. Actuators B: Chem. 160 (1) (2011) 623-631.

[24] E. Wijaya, N. Maalouli, R. Boukherroub, S. Szunerits, J. Vilcot, Graphene-based high-performance surface plasmon resonance biosensors, Proc. SPIE vol. 8424, (2012), p. 84240R.

[25] B. Song, D. Li, W. Qi, M. Elstner, C. Fan, H. Fang, Graphene on au (111): a highly conductive material with excellent adsorption properties for high-resolution bio/ nanodetection and identification, ChemPhysChem 11 (3) (2010) 585-589.

[26] M. Bruna, S. Borini, Optical constants of graphene layers in the visible range, Appl. Phys. Lett. 94 (3) (2009) 031901.

[27] R.R. Nair, P. Blake, A.N. Grigorenko, K.S. Novoselov, T.J. Booth, T. Stauber, N.M. Peres, A.K. Geim, Fine structure constant defines visual transparency of graphene, Science 320 (5881) (2008) 1308.

[28] A.J. Haes, R.P. Van Duyne, A nanoscale optical biosensor: sensitivity and selectivity of an approach based on the localized surface plasmon resonance spectroscopy of triangular silver nanoparticles, J. Am. Chem. Soc. 124 (35) (2002) 10596-10604.

[29] P.K. Jain, K.S. Lee, I.H. El-Sayed, M.A. El-Sayed, Calculated absorption and scattering properties of gold nanoparticles of different size, shape, and composition: applications in biological imaging and biomedicine, J. Phys. Chem. B 110 (14) (2006) 7238-7248.

[30] K.-S. Lee, M.A. El-Sayed, Gold and silver nanoparticles in sensing and imaging: sensitivity of plasmon response to size, shape, and metal composition, J. Phys. Chem. B 110 (39) (2006) 19220-19225.

[31] S. Zeng, K.V. Sreekanth, J. Shang, T. Yu, C.-K. Chen, F. Yin, D. Baillargeat, P. Coquet, H.-P. Ho, A.V. Kabashin, et al., Graphene-gold metasurface architectures for ultrasensitive plasmonic biosensing, Adv. Mater. 27 (40) (2015) 6163-6169.

[32] J. Leem, M.C. Wang, P. Kang, S. Nam, Mechanically self-assembled, three-dimensional graphene-gold hybrid nanostructures for advanced nanoplasmonic sensors, Nano Lett. 15 (11) (2015) 7684-7690.

[33] M.C. Sherrott, P.W. Hon, K.T. Fountaine, J.C. Garcia, S.M. Ponti, V.W. Brar, L.A. Sweatlock, H.A. Atwater, Experimental demonstration of $>230$ phase mod ulation in gate-tunable graphene-gold reconfigurable mid-infrared metasurfaces, Nano Lett. 17 (5) (2017) 3027-3034.

[34] W. Rechberger, A. Hohenau, A. Leitner, J. Krenn, B. Lamprecht, F. Aussenegg, Optical properties of two interacting gold nanoparticles, Opt. Commun. 220 (1) (2003) 137-141.

[35] J. Goffard, D. Gérard, P. Miska, A.-L. Baudrion, R. Deturche, J. Plain, Plasmonic engineering of spontaneous emission from silicon nanocrystals, Sci. Rep. 3 (2013).

[36] M.S. Rahman, M.S. Anower, M.R. Hasan, M.B. Hossain, M.I. Haque, Design and numerical analysis of highly sensitive Au-MoS2-graphene based hybrid surface plasmon resonance biosensor, Opt. Commun. 396 (2017) 36-43.

[37] Y. Kaya, S. Ayas, A.E. Topal, H. Guner, A. Dana, Sensitivity comparison of localized plasmon resonance structures and prism coupler, Sens. Actuators B: Chem. 191 (2014) 516-521. 